\title{
Gasdynamic Simulation of Aerosol Deposition Method
}

\author{
Hiroshi Katanoda ${ }^{1}$ and Kazuyasu Matsuo ${ }^{2}$ \\ ${ }^{1}$ Department of Mechanical Engineering, Kagoshima University, 1-21-40 Korimoto, Kagoshima 890-0065, Japan \\ ${ }^{2}$ Department of Mechanical Systems and Environmental Engineering, The University of Kitakyushu, Kitakyushu 808-0135, Japan
}

\begin{abstract}
The gas flow of aerosol deposition method (ADM) is simulated using the method of computational fluid dynamics (CFD), and the velocity of particles flowing in the gas flow is also calculated by numerically integrating Newton's second law of motion. The process gas is nitrogen and the particle material is $\mathrm{Pb}(\mathrm{Zr}, \mathrm{Ti}) \mathrm{O}_{3}(\mathrm{PZT})$. The calculated particle velocities are compared with experimental results obtained by using a slit-cell method (SCM), which may disturb the gas-particle jet-flow of ADM during the measurement of particle velocity. The comparison of calculated and measured results reveals that the SCM can cause unexpected measurement error due to disturbing the gas-particle jet-flow caused by the stagnation pressure exceeding a certain value. [doi:10.2320/matertrans.47.1620]
\end{abstract}

(Received November 30, 2005; Accepted March 24, 2006; Published July 15, 2006)

Keywords: aerosol deposition method, fluid dynamics, numerical simulation, under-expanded impinging jet, particle velocity

\section{Introduction}

The application of piezoelectric ceramics is an indispensable technology to make devices for micro electro mechanical systems such as sensors or actuators. Among traditional dry coating processes, plasma spraying has been widely used to deposit ceramics particles. The plasma spray gun, however, consumes a large amount of electric energy to produce a plasma flow into which ceramics particles are injected. In addition to that, the substrate needs to be heat-resistant to keep its material properties and size unchanged after the exposure to a high-temperature plasma-particle flow. On the other hand, aerosol deposition method ${ }^{1)}$ (ADM) uses helium or nitrogen as a process gas at room temperature and needs no additional energy to heat both the process gas and ceramics particles. The amount of heat energy consumed in the process or transferred to the substrate by ADM is negligibly small compared to that by plasma spraying.

\section{Aerosol Deposition Method}

Figure 1 shows a schematic diagram of ADM. The gas flow mixed with ceramics particles smaller than around $2 \mu \mathrm{m}$ is supplied to a nozzle which has a converging round or rectangular cross section. The nozzle discharges the gasparticle flow towards a substrate in a vacuum chamber, where the gas pressure is lowered in the range between several hundred $\mathrm{Pa}$ and several $\mathrm{kPa}$ to make a coating. According to one-dimensional isentropic theory, ${ }^{2)}$ the stagnation pressure of more than twice the pressure in the vacuum chamber (back pressure) means that the Mach number of the gas flow at the nozzle exit is unity for a converging nozzle when helium or nitrogen is used as a process gas. In ADM, the stagnation pressure upstream of the nozzle is more than ten times the back pressure. In this case, the gas velocity reaches the speed of sound at the nozzle exit. Then, the gas flow expands to supersonic speed through expansion waves originated at the lip of the nozzle exit. When the jet flow is supersonic just upstream of the substrate, the flow decelerates to subsonic speed through a normal shock wave. In the field of gasdynamics, which is the authors' interest, the gas flow of

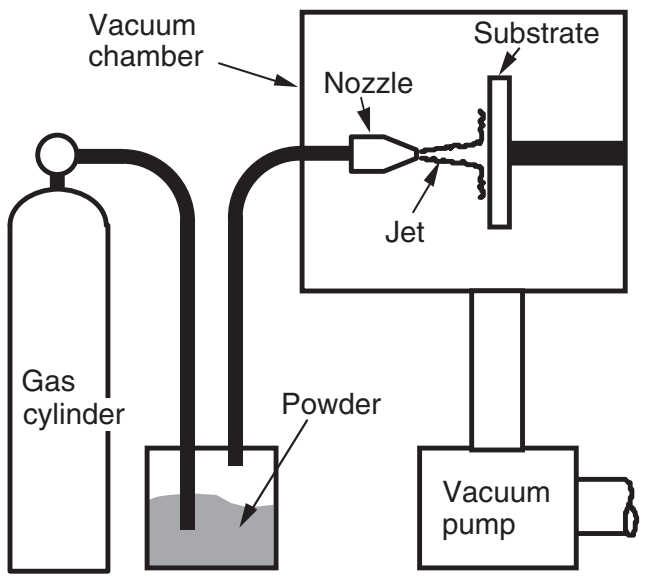

Fig. 1 Schematic diagram of ADM.

this type is called an under-expanded impinging jet. Such a kind of jet flow has been extensively studied in the past, ${ }^{3,4)}$ however, mostly restricted to turbulent flow which can not be applied to laminar flow of ADM. Therefore, the gasdynamic research of $\mathrm{ADM}$ is essential for the understanding and the improvement of the process.

Almost all of the experimental work ${ }^{1,5,6)}$ of ADM has been conducted by a research group of the National Institute of Advanced Industrial Science and Technology in Japan from a view point of materials science. The material properties, such as optical transmittance and piezoelectric properties, of the ceramics coatings are reported in several papers. The ADM seems to be an attractive technology in the field of dry coating processes. However, the mechanism of how ceramics particles deposit on a substrate has not been clarified yet. According to the experimental result, the impact velocity of particles onto the substrate is one of the important parameters to control coating properties. In the field of fluid dynamics, a non-contact measurement method, such as laser Doppler velocimetry ${ }^{7)}$ or particle image velocimetry, ${ }^{8)}$ are normally used to measure particle velocity. However, such a noncontact measurement method is difficult to apply to the ADM because the process is conducted in a vacuum chamber, not in 
an open space. Instead of a non-contact measurement method, Lebedev, et al. ${ }^{5)}$ invented a simple contact method to measure an average particle velocity. In this paper, the method is called slit-cell method (SCM). In the SCM, which is described in the next chapter in detail, a cell with a slit transverses the gas-particle jet. The cell crossing the jet is assumed not to disturb the jet flow. So far, however, the extent to which the cell disturbs the jet flow and possibly causing a measurement error in the particle velocity has not been investigated yet.

In the present work, the gas flow of ADM is simulated by using the method of computational fluid dynamics (CFD) to clarify how the gas flows through the nozzle to the substrate. Numerical calculations using mass-averaged Navire-Stokes equations are applied to two-dimensional gas flow of the ADM operated at standard stagnation pressures upstream of the nozzle. The particle velocities are also calculated by numerically integrating Newton's second law of motion. Then, simulated particle velocities are compared to experimental data. The comparison reveals that the SCM can cause unexpected measurement error due to disturbing the highspeed jet flow caused by the stagnation pressure when exceeding a certain value.

\section{Background}

In this paper, experimental data ${ }^{6)}$ obtained by a rectangular nozzle with nitrogen as a process gas are used to compare calculations. The experimental configuration and test conditions are briefly described in this chapter to understand the results of comparison between the experimental and numerical velocities of the particle.

Figure 2 shows a close view of the impinging jet included in Fig. 1. The nozzle exit has a height of $0.4 \mathrm{~mm}$. The gas jet containing particles of $\mathrm{Pb}(\mathrm{Zr}, \mathrm{Ti}) \mathrm{O}_{3}$ (PZT) is discharged from the nozzle towards the substrate. The distance between the nozzle exit and the substrate (stand-off distance) is less than $20 \mathrm{~mm}$. In Fig. 2, of course, the impinging jet is not disturbed by any obstacles used to measure the particle velocity.

Figures 3 shows how the SCM works. This method uses a cell moving perpendicular to the jet flow at a velocity $W$ as shown in Fig. 3(a). The cell has a substrate on the right side and a plate with a slit on the left side. The slit-cell goes across

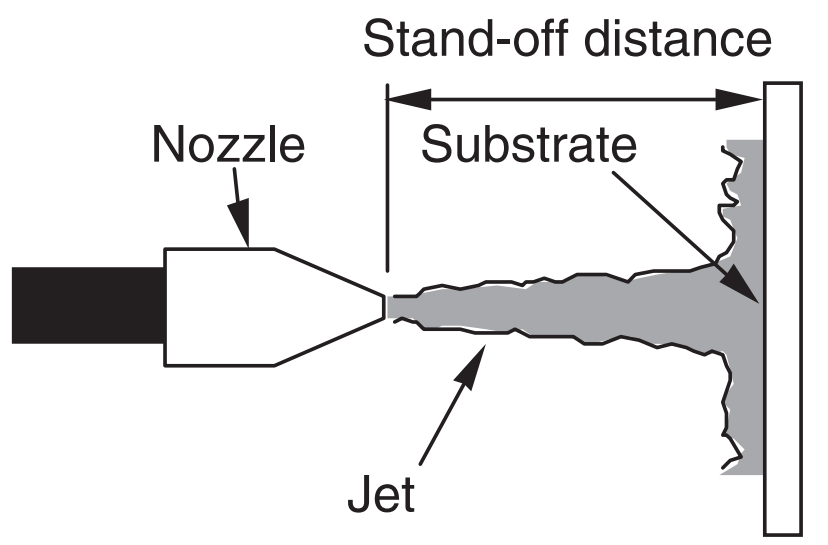

Fig. 2 Jet flow of ADM.

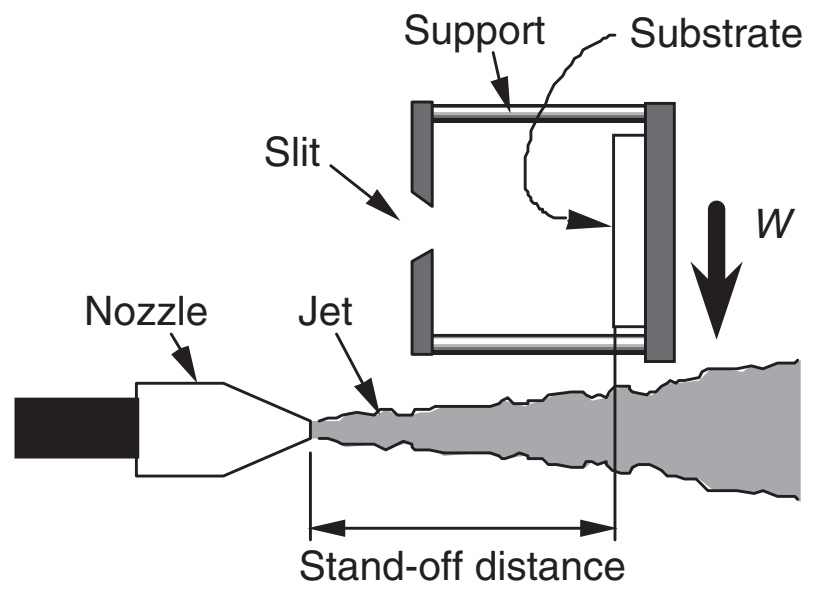

(a) Before crossing the jet

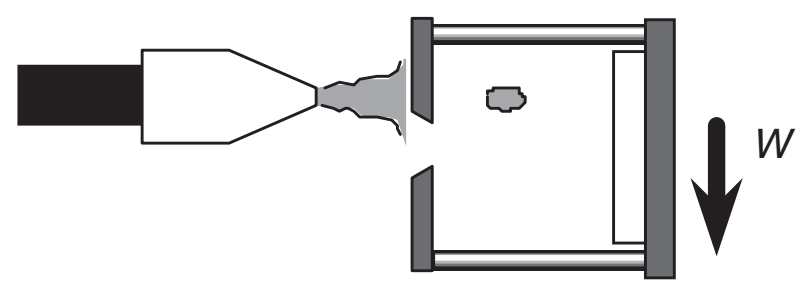

(b) While crossing the jet

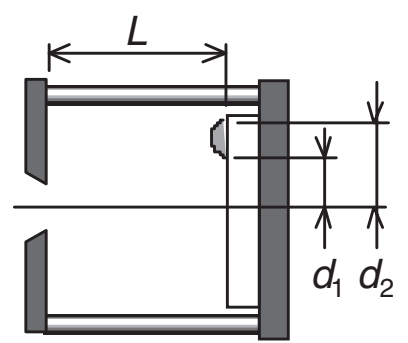

(c) After crossing the jet

Fig. 3 Particle-velocity measurement of ADM with SCM.

the jet as shown in Fig. 3(b) to cut the stream, creating a small projectile of gas and particle inside the cell. Then, after a fraction of a second, the projectile collides with the substrate as shown in Fig. 3(c). By measuring the distances $d_{1}$ and $d_{2}$ from the center line, the average particle velocity $\bar{u}_{p}$ over the distance $L$ is estimated as

$$
\bar{u}_{p}=\frac{W L}{\left(d_{1}+d_{2}\right) / 2}
$$

where, $L$ is the distance between the slit and the substrate. The particle velocity varies from nozzle exit to the substrate depending on the local gas velocity and density. But eq. (1) calculates an average value over the distance $L$. According to Ref. 5), measured particle velocity of PZT under typical spray conditions does not depend on the distance range of $L=3-25 \mathrm{~mm}$. The powder used in ADM includes particles of a certain range of diameter, for example $0.1-2 \mu \mathrm{m}$. Among them, only particles with a particular range of diameter can be deposited on the substrate. That is, particles that are too small or too big are not coated on the substrate. Therefore, the method can measure a kind of average particle-velocity over 
the distance $L$ and over the particular range of diameters which can be coated. The maximum measurement uncertainty of this method is reported as $7 \%^{5)}$ due to the accuracy of measuring $d_{1}$ and $d_{2}$ and the uncertainty of the spreading angle of particle flow. An additional error caused by disturbing the jet flow is not taken into account in Ref. 5). In this measurement method, the slit-cell, which crosses the jet, is supposed to cause no disturbance to the jet flow. Accordingly, we need to keep in mind that the SCM will give a proper value of average particle velocity under the assumption that the slit-cell does not disturb the impinging jet.

\section{Numerical Calculations}

\subsection{Gas flow}

The cross-sectional area at the exit of the nozzle used in Ref. 6) is $0.4 \mathrm{~mm} \times 10 \mathrm{~mm}$, and the aspect ratio is as large as $10 / 0.4=25$. So, the flow is assumed to be two-dimensional in the CFD model. The governing equations of the gas flow are given by the conservation form of the two-dimensional, time-dependent Navier-Stokes equations along with the equation of state. The Reynolds number $R_{e}$ at the nozzle exit with an exit-height of $0.4 \mathrm{~mm}$ as a length scale is less than 640. According to Troutt and McLaughlin ${ }^{9)}$ the jet flow is laminar for $R_{e}<1000$. Therefore, no turbulence model is employed. The governing equations are solved sequentially in an implicit, iterative manner using a finite difference formulation. For the present calculations, the governing equations are solved with the Chakravarthy-Osher type thirdorder, upwind, total variation diminishing scheme for the convective terms. A second-order, central difference scheme is used for the diffusive terms. The stagnation pressure upstream of the nozzle, $p_{o s}$, was set at constant values from 1 to $10 \mathrm{kPa}$ in the CFD model. The back pressure was fixed at $100 \mathrm{~Pa}$. The stagnation temperature of nitrogen gas both upstream of the nozzle and outside the nozzle was set at $300 \mathrm{~K}$.

The computational domain for the ADM is shown in Fig. 4. The thick solid line shows the boundary of the computational domain. The nitrogen gas is discharged from a converging nozzle towards the substrate. The stand-off distance is $15 \mathrm{~mm}$. The solid-wall boundary-condition is used along the surface of the substrate.

Figure 5 shows the computational domain for the ADM with SCM. The computational domain is the upper half of the flow field. This is because the simulated results of the ADM were almost symmetrical about the center line of the jet flow (Fig. 4). Accordingly, the same was expected for the ADM with SCM (Fig. 5). In the experiment ${ }^{1)}$ of the SCM, the transverse velocity of the cell is around $1 / 10$ of the jet velocity $(322 \mathrm{~m} / \mathrm{s})$ at the nozzle exit. Therefore, the authors regard the cell to be stationary compared to the jet velocity to simplify the computation. The slit is located $5 \mathrm{~mm}$ downstream of the nozzle exit, and the distance $L$, explained in Fig. 3(c), is $10 \mathrm{~mm}$. The slit wall is infinitesimally thin in the CFD model. The slit has $0.8 \mathrm{~mm}$-height aligned with the center line of the nozzle. The stand-off distance is $15 \mathrm{~mm}$, the same as the ADM.

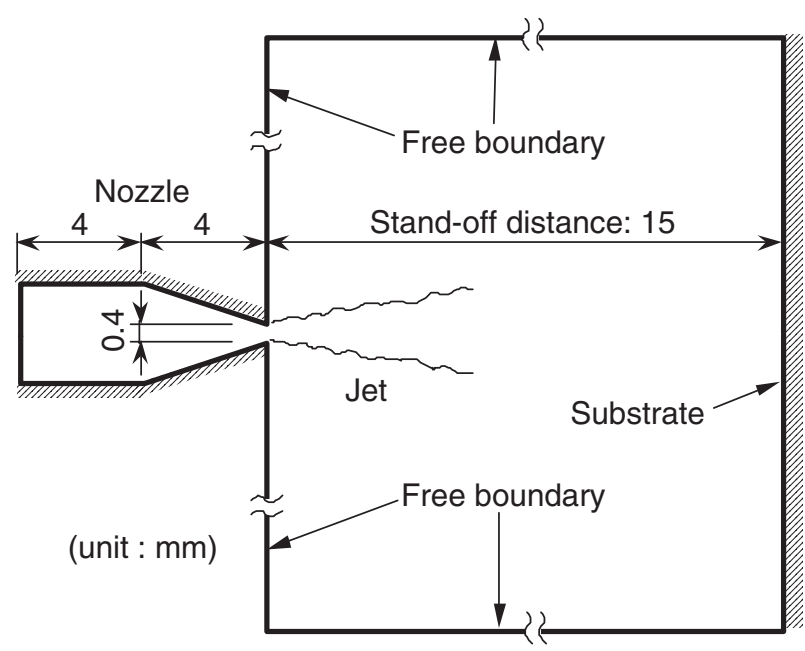

Fig. 4 Computational domain for ADM.

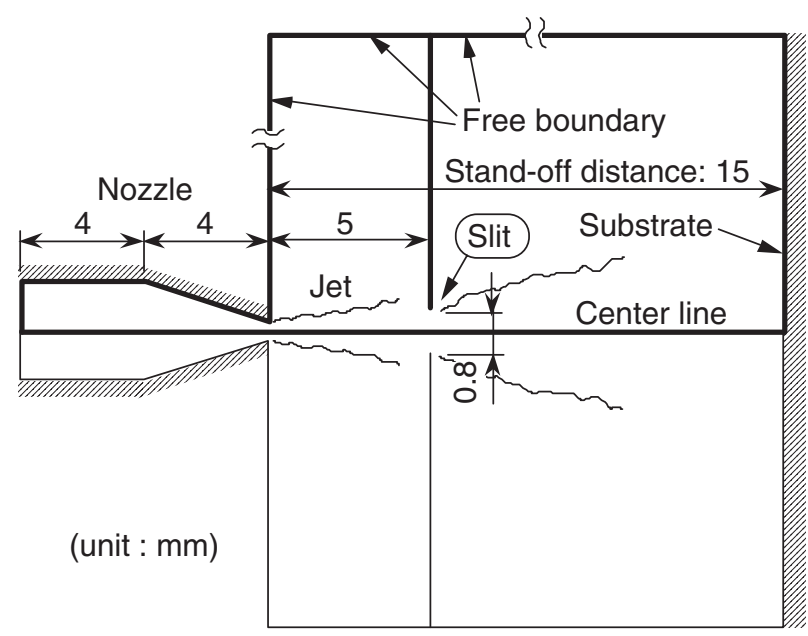

Fig. 5 Computational domain for ADM + SCM.

\subsection{Particle flow}

To describe the particle motion, we use the Lagrangian formulation. The following assumptions are made to simplify the analysis.

- The particles are sphere in shape.

- The interaction between particles can be ignored.

- The only force acting on a particle is drag force.

- The presence of particles has a negligible effect on the gas velocity and temperature field.

- The particles have a constant specific heat and a constant density.

- The temperature in the particle is uniform.

As a result of the assumptions presented above, the gas-solid two-phase problem can then be independently solved. One can simulate the gas flow first, then use the resulting thermal and velocity fields to study the flow of different particles.

The particle velocities are determined from a step-wise integration of their equations of motion under the influence of gasdynamic drag force. In this paper, the particle motion along the center line is calculated. The governing equation for momentum transfer between a single particle of mass $m_{p}$ and a gas can be written as 
(a) $\mathrm{ADM}$

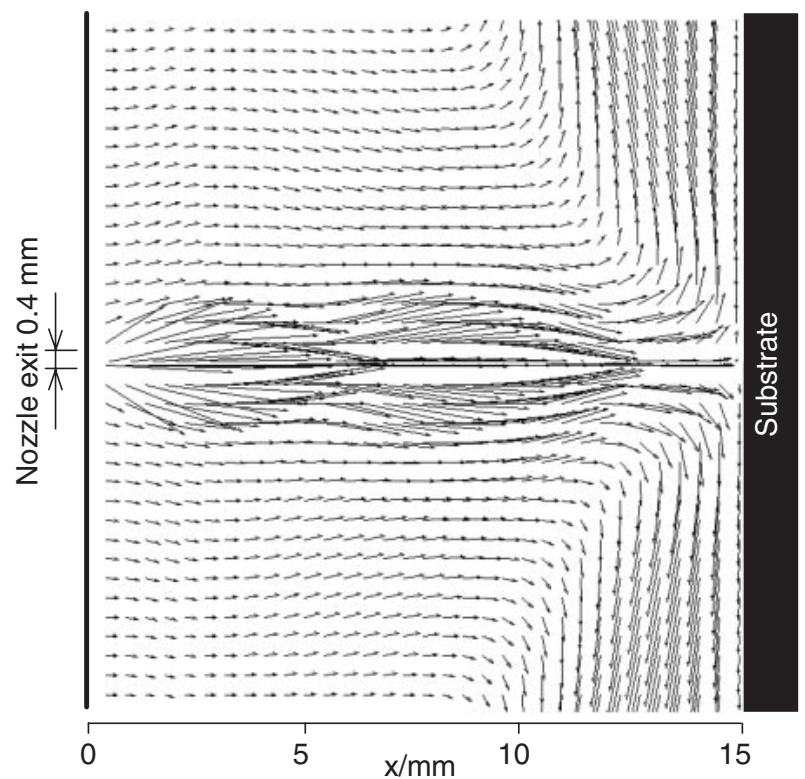

(b) $\mathrm{ADM}+\mathrm{SCM}$

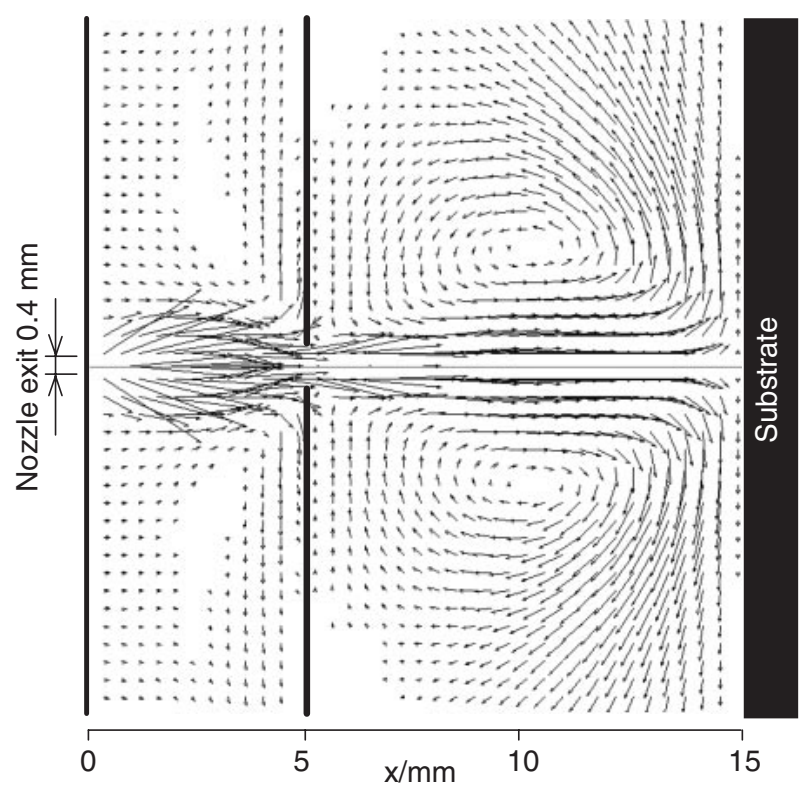

Fig. 6 Simulated velocity vector for $p_{o s}=2 \mathrm{kPa}$.

$$
m_{p} \frac{d u_{p}}{d t}=\frac{1}{2} c_{d} \rho_{g}\left(u_{g}-u_{p}\right)\left|u_{g}-u_{p}\right| A_{p}
$$

where, $u_{p}$ the particle velocity, $u_{g}$ the gas velocity, $\rho_{g}$ the gas density, $t$ the time, $c_{d}$ the drag coefficient of the particle and $A_{p}$ the projected area of the particle. The drag coefficient, $c_{d}$, is given by the equations proposed by Henderson. ${ }^{10)}$ The particle temperatures are also calculated by solving the unsteady heat-transfer equation of a sphere particle flowing in a gas flow. Based on the measurement of Ref. 1), the diameter of the PZT particle is taken as $0.2 \mu \mathrm{m}$ in this report.

\section{Results and Discussion}

Figure 6 shows simulated velocity vector of gas flow in the (a) ADM

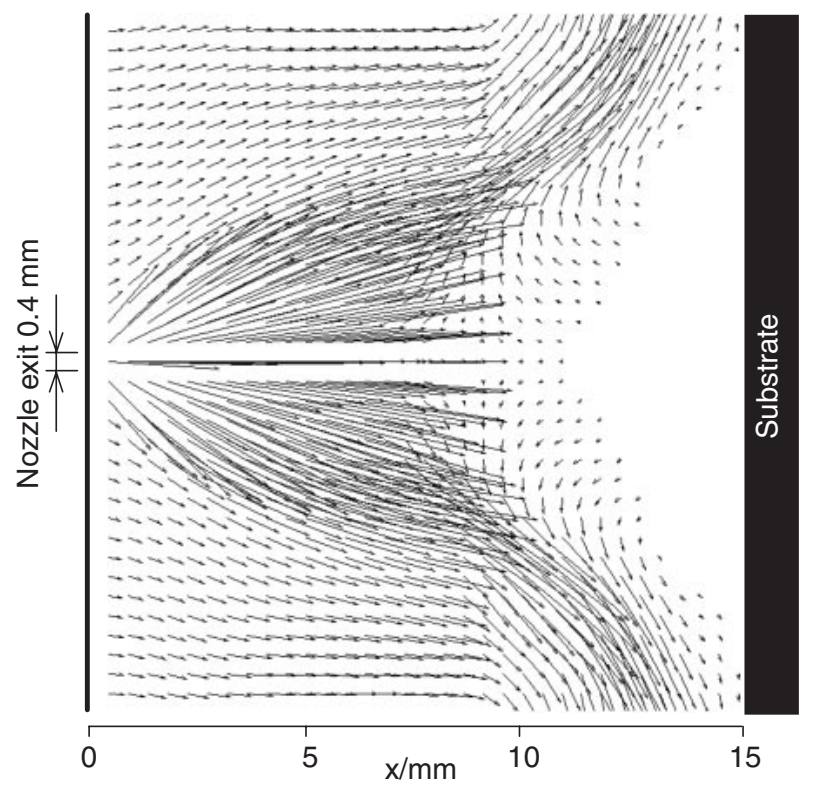

(b) $\mathrm{ADM}+\mathrm{SCM}$

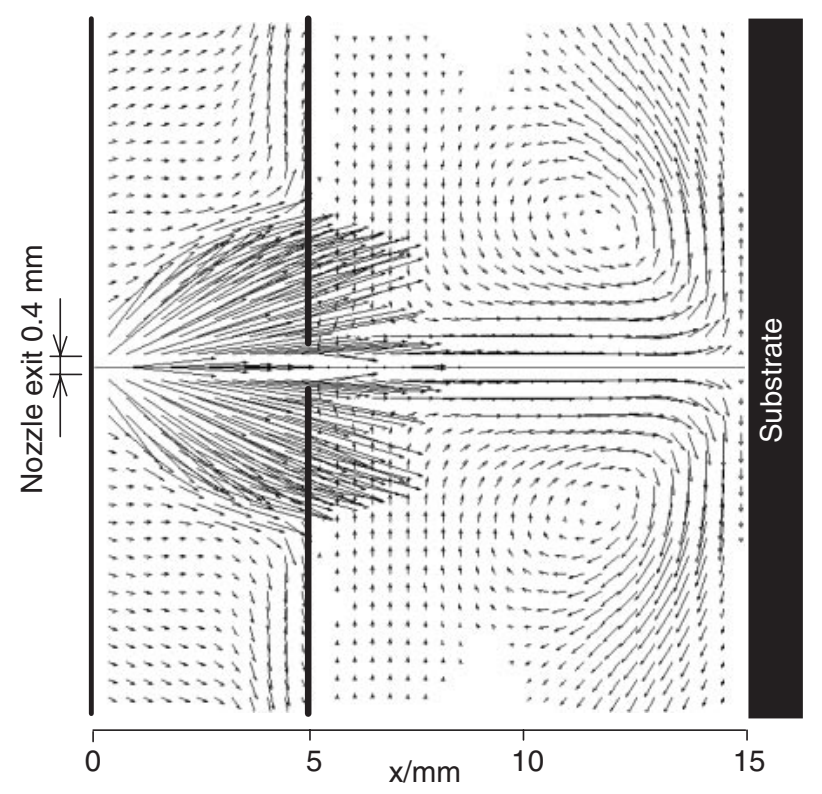

Fig. 7 Simulated velocity vector for $p_{o s}=6 \mathrm{kPa}$.

vacuum chamber at $p_{\text {os }}=2 \mathrm{kPa}$ for ADM (a) and ADM with SCM (b). The gas flow in the nozzle is not shown in the figure. The length of the arrows in the figure is linearly dependent on the magnitude of the gas velocity. The axial distance from the nozzle exit, $x$, is shown at the bottom of each figure. For the ADM, Fig. 6(a), the gas expands into the vacuum chamber to reach the substrate and flows away along the substrate. For the ADM with SCM, Fig. 6(b), the central part of the jet flows through the slit and reaches the substrate. A pair of vortices is formed outside the jet flow between the slit and the substrate.

Figure 7 shows simulated velocity vector of gas flow at $p_{o s}=6 \mathrm{kPa}$ for ADM (a) and ADM with SCM (b). For the ADM, Fig. 7(a), the gas is strongly accelerated from the nozzle exit to the vacuum chamber due to larger stagnation 
(a) $\mathrm{ADM}$

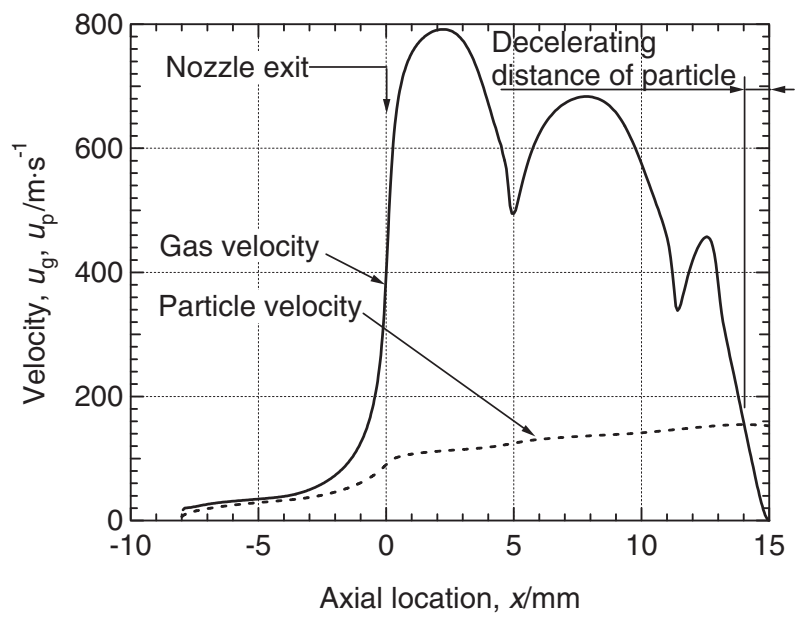

(b) $\mathrm{ADM}+\mathrm{SCM}$

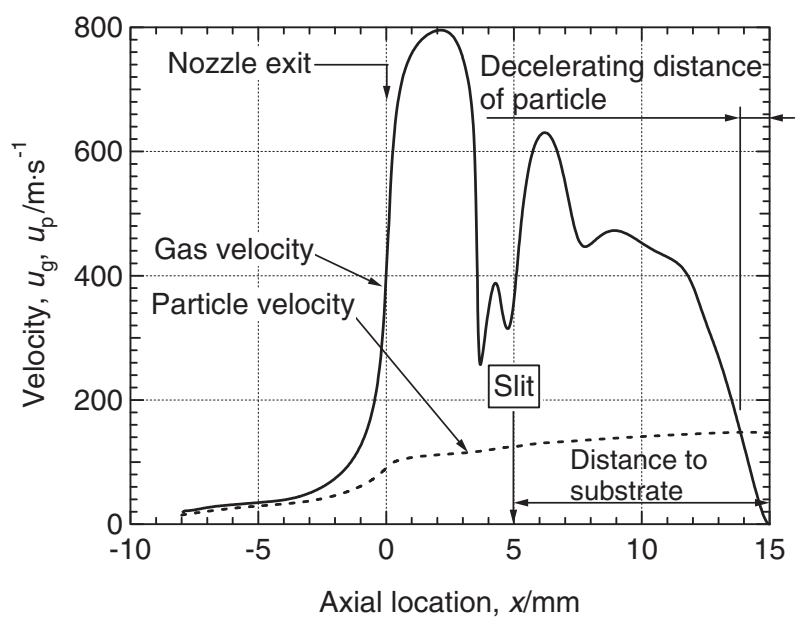

Fig. $8 \mathrm{Gas} /$ particle velocity on center line for $p_{o s}=2 \mathrm{kPa}$.

pressure $p_{\text {os }}$ compared to that for Fig. 6. The notable peculiarity of the flow is that the reverse flow from right to left in the figure is generated in the downstream region, which decelerates the particle as shown later. For the ADM with SCM, Fig. 7(b), the acceleration of the gas flow is blocked by the slit-wall and a small part of gas from the nozzle goes through the slit. As a result, no reverse flow is generated along the center line between the slit and the substrate. A large number of velocity vector arrows go through the slit-wall in Fig. 7(b). It means that the high-speed gas flow impinges on the upstream-side of the slit-wall, not penetrating the slit-wall.

Calculated particle velocity as well as the gas velocity along the center line of Fig. 6 is shown in Fig. 8 for ADM (a) and ADM with SCM (b). The vertical axis shows gas/particle velocity. The horizontal axis shows the axial distance measured from the nozzle exit along the center line in the downstream direction. The solid line and the dotted line in Fig. 8 show gas velocity and particle velocity, respectively. For the ADM, Fig. 8(a), the gas is sharply accelerated at the nozzle exit to reach the speed of sound $(322 \mathrm{~m} / \mathrm{s})$. Then, the gas flow repeats acceleration and deceleration in the downstream direction until the gas flow impinges on the substrate. The PZT particle is accelerated in the gas stream by the (a) ADM

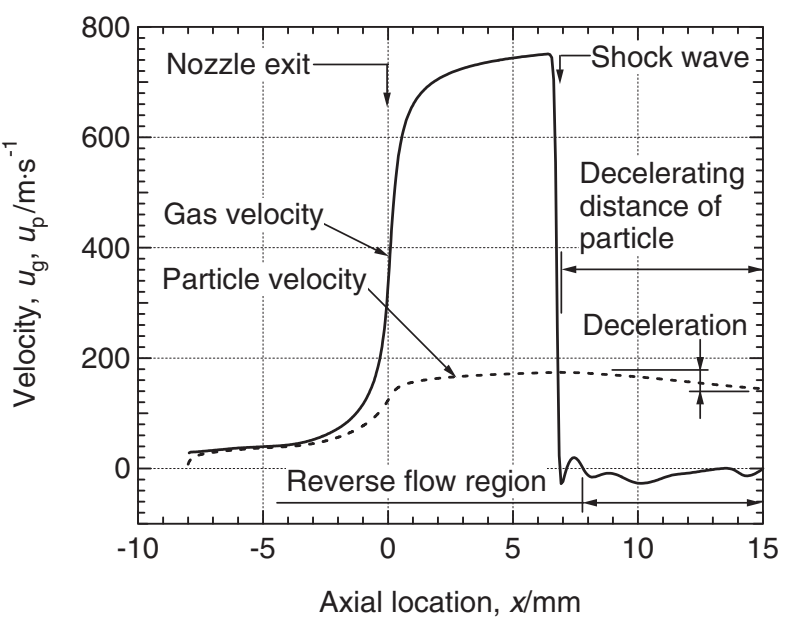

(b) $\mathrm{ADM}+\mathrm{SCM}$

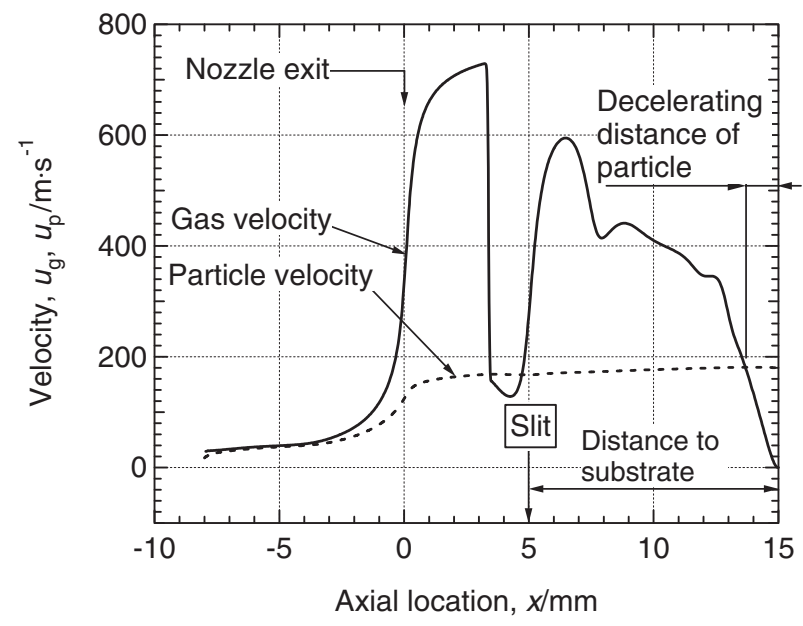

Fig. $9 \mathrm{Gas} /$ particle velocity on center line for $p_{o s}=6 \mathrm{kPa}$.

gasdynamic drag force. The acceleration of the particle is moderate in the jet flow due to the low density of the gas. In the region of $x=14-15 \mathrm{~mm}$, the particle velocity is larger than the gas velocity; the particle is decelerated in this region. However, the extent of deceleration of the particle is negligibly small due to the small gas density and short decelerating distance.

For ADM with SCM, Fig. 8(b), the gas flow along the center line is altered from the ADM jet for $3 \mathrm{~mm}<x<$ $13 \mathrm{~mm}$ due to the insertion of the slit. Nonetheless, the particle velocity is almost unchanged from the undisturbed case of Fig. 8(a). Therefore, it is confirmed that the insertion of the slit in the ADM jet has a negligible effect on the particle velocity for $p_{o s}=2 \mathrm{kPa}$.

The particle velocity as well as the gas velocity along the center line of Fig. 7 is shown in Fig. 9 for ADM (a) and ADM with SCM (b). For the ADM, Fig. 9(a), the gas velocity abruptly drops at $x=6.5 \mathrm{~mm}$. According to the Mach number distribution along the center line, not shown in this paper, the abrupt drop in the gas velocity is caused by a strong normal shock wave generated at $x=6.5 \mathrm{~mm}$. The gas velocity drops from supersonic to subsonic speed through the normal shock wave. Between the shock wave and the substrate $(6.5 \mathrm{~mm}<x<15 \mathrm{~mm})$, the gas velocity remains 


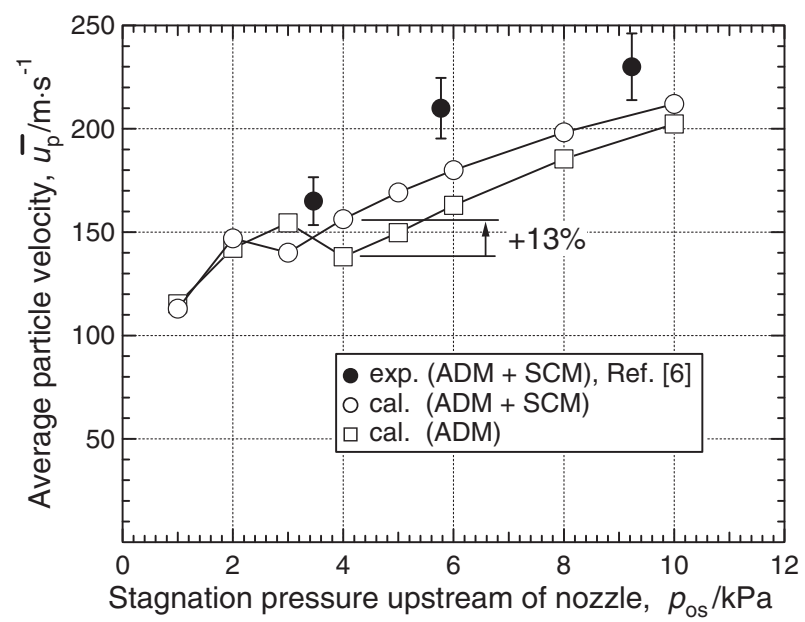

Fig. 10 Average particle velocity versus stagnation pressure upstream of nozzle.

smaller than the particle velocity and even a reverse flow is generated. That is, the particle flies against the decelerating drag force, decreasing its velocity in the distance of $6.5 \mathrm{~mm}<x<15 \mathrm{~mm}$.

As for the ADM with SCM, Fig. 9(b), the gas is suddenly decelerated upstream of the slit at $x=3 \mathrm{~mm}$. Then, the gas starts acceleration again through the slit in the downstream direction. After reaching the second peak at $x=6.5 \mathrm{~mm}$, the gas velocity goes down to the sonic speed at $x=12 \mathrm{~mm}$, then it becomes stagnant on the substrate at $x=15 \mathrm{~mm}$. The particle continues acceleration until it reaches the gas velocity at $x=13 \mathrm{~mm}$. Then, the particle is decelerated until it hits the substrate. Because of the smaller decelerating distance of the particle for Fig. 9(b) than that for Fig. 9(a), the extent of the deceleration of the particle velocity is almost zero for Fig. 9(b). From Fig. 9 it is confirmed that the insertion of the slit excessively smears the normal shock wave, resulting in the increase in particle velocity for the ADM with SCM compared to the ADM.

Figure 10 summarizes the comparison between the calculated and measured average particle velocities. In the figure, the solid circle denotes the average particle velocity measured by the SCM. The open symbols mean simulated results; open circle for ADM with SCM and open square for ADM. Because the SCM measures an averaged particle velocity over the distance $L$ as shown in Fig. 3, all the simulated values in Fig. 10 are also averaged over the distance $L=10 \mathrm{~mm}$. In Fig. 10, the simulated results for ADM with SCM (open circle) reasonably agree with the experimental results (solid circle) measured by the SCM. It means that the present calculation of the particle velocity as well as the impinging jet with slit simulates the SCM well. The deviation of the simulated velocities from the experimental values may lie in the simplification of the twodimensional jet flow and the stationary slit-cell in the CFD model.

Finally, the authors try to estimate the maximum deviation in the SCM by comparing the simulated particle velocities in Fig. 10. The simulated particle velocities for ADM (open square) are smaller than ADM with $\mathrm{SCM}$ for $p_{\text {os }} \geq 4 \mathrm{kPa}$. The maximum deviation of the particle velocity caused by the SCM is about $+13 \%$ at $p_{\text {os }}=4 \mathrm{kPa}$. The deviation occurs by smearing the normal shock wave in the jet due to the insertion of the slit. The deviation decreases by increasing $p_{o s}$ for $p_{o s} \geq 4 \mathrm{kPa}$.

\section{Conclusions}

The extent of the measurement error in the slit-cell method (SCM) used to measure the particle velocity for the aerosol deposition method (ADM) was estimated by numerical simulation. The gas flow was assumed to be two-dimensional and the slit-cell was regarded to be stationary against the converging nozzle. Nitrogen was used as a process gas. The simulation was performed for the ADM and ADM with SCM. The latter case simulates the SCM which is used to experimentally measure the particle velocity. The results of the simulation are summarized as follows:

(1) The simulated particle velocities for ADM with SCM agree well with the experimental values obtained by the SCM.

(2) When the slit is not inserted in the impinging jet, a strong normal shock wave is generated in front of the substrate for $p_{o s} \geq 4 \mathrm{kPa}$. The gas velocity between the normal shock wave and the substrate is much smaller than the particle velocity causing the deceleration of the particle.

(3) The normal shock wave which is generated for $p_{o s} \geq$ $4 \mathrm{kPa}$ is excessively smeared when the slit-cell is inserted in the impinging jet. As a result, the gas flow keeps larger velocity than the particle, until close to the substrate. As a result, the extent of deceleration of the particle becomes negligibly small, with the SCM larger particle velocity is obtained compared to the ADM.

(4) With the SCM, the maximum deviation of the particle velocity due to disturbing the jet flow by inserting the slit is about $+13 \%$ at $p_{o s}=4 \mathrm{kPa}$.

\section{REFERENCES}

1) J. Akedo and M. Lebedev: Jpn. J. Appl. Phys. 33 (1999) 5397-5401.

2) H. W. Liepmann and A. Roshko: Elements of Gas-dynamics, Dover Publications, Inc., New York (2001) pp. 39-61.

3) C. D. Donaldson and R. S. Snedeker: J. Fluid Mech. 45 (1971) 281319.

4) J. C. Carling and B. L. Hunt: J. Fluid Mech. 66 (1974) 159-176.

5) M. Lebedev, J. Akedo, M. Mori and T. Eiju: J. Vac. Sci. Technol. A 18 (2000) 563-566.

6) J. Akedo and M. Lebedev: Jpn. J. Appl. Phys. 40 (2001) 5528-5532.

7) J. C. Lau, P. J. Morris and M. J. Fisher: J. Fluid Mech. 93 (1979) 1-27.

8) R. Elavarasan and L. Venkatakrishnan: J. Visualization 2 (2000) 213221.

9) T. R. Troutt and D. K. McLaughlin: J. Fluid Mech. 116 (1982) 123156.

10) C. B. Henderson: AIAA J. 14 (1976) 707-708. 